\title{
Effects of level and type of energy source (volatile fatty acids or glucose) on milk yield, composition and coagulating properties in dairy cows
}

\author{
Catherine Hurtaud*, Henri Rulquin, Raymond Vérité \\ Station de recherches sur la vache laitière, Inra, 35590 Saint-Gilles, France
}

(Received 18 July 1997; accepted 23 March 1998)

\begin{abstract}
Four fistulated Holstein cows were arranged in a $4 \times 4$ Latin square design to study the effects of level and type of energy source on milk yield and composition. Treatments consisted of a basal diet fed alone (low energy treatment) or with 3.3 Mcal of net energy for lactation from extra nutrients perfused either into the rumen (either propionic acid or a mixture of volatile fatty acids) or into the duodenum (glucose). Increasing the energy input without changing the volatile fatty acid profile improved milk yield and slightly increased milk protein and fat yields. Compared with the isoenergetic mixture of volatile fatty acids, both propionic acid and glucose infusions significantly decreased fat content $(-4.5 \mathrm{~g} / \mathrm{kg}$ ) and yields (respectively, -111 and -160 $\mathrm{g} / \mathrm{d}$ ), but affected fatty acid proportion and yield differently (more elongation process and less C18 with glucose infusion). Protein yield was slightly increased by propionic acid infusion but not by glucose because of the counterbalanced effects on milk yield $(-1.3 \mathrm{~kg} / \mathrm{d})$ and protein content $(1.5 \mathrm{~g} / \mathrm{kg})$. The coagulating properties of milk were directly linked to variations in protein, casein and mineral contents. In conclusion, propionic acid or glucose scarcely affected milk protein content, but induced a similar decrease in milk fat content probably through different metabolic pathways. ( $\mathrm{Inra/Elsevier,} \mathrm{Paris}$
\end{abstract}

milk composition / milk coagulation / VFA infusion / propionic acid infusion / glucose infusion

Résumé - Effets du niveau et de la nature de l'énergie (AGV ou glucose) sur la production, la composition et l'aptitude à la coagulation du lait. Les effets du niveau et de la nature de l'énergie sur la production et la composition du lait ont été étudiés au cours d'un essai mené avec quatre vaches fistulées selon un schéma en carré latin $4 \times 4$. Les vaches recevaient soit le régime de base seul (témoin négatif), soit supplémenté avec 3,3 Mcal d'énergie nette lait sous forme de nutriments perfusés dans le rumen (mélange d'acides gras volatils ou acide propionique) ou dans le duodénum (glucose). L'augmentation du niveau énergétique sans modification du profil

\footnotetext{
* Correspondence and reprints

E-mail: hurtaud@st-gilles.rennes.inra.fr
} 
fermentaire a entraîné une augmentation de la production laitière et des productions de matières grasses et de protéines. En comparaison avec le mélange isoénergétique d'acides gras volatils, les infusions d'acide propionique et de glucose ont provoqué une chute importante du taux butyreux $(-4.5 \mathrm{~g} / \mathrm{kg}$ ) et des productions de matières grasses (respectivement $-111 \mathrm{et}-160 \mathrm{~g} / \mathrm{j}$ ), mais ont affecté de manière différente la composition en acides gras du lait (quantité plus importante des acides gras moyens et moins d'acides gras en $\mathrm{C} 18$ avec le glucose). La production de protéines a été légèrement augmentée par l'infusion d'acide propionique, mais non par l'infusion de glucose à cause de l'effet opposé sur le taux protéique $(+1,5 \mathrm{~g} / \mathrm{kg})$ et la production laitière $(-1,3 \mathrm{~kg} / \mathrm{j})$. L'aptitude du lait à la coagulation reflétait les modifications de composition en protéines, en caséines et en minéraux. En conclusion, l'acide propionique et le glucose n'ont pas affecté de façon sensible la synthèse des protéines du lait mais ont entraîné une diminution similaire du taux butyreux probablement par des voies métaboliques différentes. (ㅇ Inra/Elsevier, Paris

\section{composition du lait / coagulation du lait / AGV / acide propionique / glucose}

\section{INTRODUCTION}

Adjustments to the diets of dairy cows can be one of the quickest ways to improve milk quality, especially the protein content and cheese-making suitability required by the processing industry. According to Coulon and Rémond [7], using concentrates to increase the energy level of the diet also provides an increase in milk protein content $(0.3 \mathrm{~g} / \mathrm{kg}$ for each Mcal of $\mathrm{NE}_{\mathrm{L}}$ ) in multiparous cows in early lactation. Several mechanisms may be involved because concentrate supplementation may increase 1) ATP necessary for protein synthesis, 2) glucose supply (ruminal propionic fermentation or by-pass starch) that spares glucogenic AA [40], or 3) AA supply that is induced by the greater bacterial synthesis associated with the increase in rumen-fermented OM [17].

Increased AA caused by greater bacterial synthesis (item 3 ) can be avoided by infusing energy as VFA into the rumen $[5,28,33,34]$ or glucose into the duodenum $[6,29]$. Unfortunately, in most of these experiments, the effects of level (item 1) and type of energy (item 2) were confounded (see review of Rulquin and Hurtaud [36]). Indeed, except for Holter et al. [13], Hurtaud et al. [14] with propionic acid (C3) infusion and for Vik-Mo et al.
[41], Clark et al. [6], Ørskov et al. [29] and Lemosquet et al. [24] with glucose infusion, comparisons of treatments were not isoenergetic because, when VFA and glucose were infused, the total energy supply (diet and infusion) was increased. The effect on milk protein yield varies in relation to the type and level of additional energy supplied. Milk protein yield often increased with infusion of $\mathrm{C} 3$ except in isoenergetic situations $[10,13,14]$ and with glucose infusion except for Clark et al. [6] and Oldick et al. [27]. In most cases, energy supplementation, either in the form of $\mathrm{C} 3$ or glucose, is followed by a decrease in butter fat content $[4,5,6,10,11,13$, $14,33,34,42]$. However, only one comparison [10] has been made between glucose and $\mathrm{C} 3$ intravenous infusions. In this experiment, C3 and glucose infusions had no effect on milk protein synthesis. The effects of $\mathrm{C} 3$ and glucose have often been confounded in glucogenic theories (sparing effect of amino acids). In fact, the mechanisms by which C3 and glucose function in reducing milk fat synthesis and in regulating protein secretion are quite unknown even if the effect of the two nutrients seemed to be the same on fat content.

This experiment was designed to assess the specific effects of energy supplemen- 
tation and type of energy on the synthesis of milk components, on the mechanisms of their syntheses (as reflected by blood metabolites), and on the cheesemaking suitability of the milk. A treatment that was deficient in energy was compared with three other treatments that provided equal amounts of energy in the form of either a mixture of VFA or $\mathrm{C} 3$ infused into the rumen or of glucose infused into the duodenum.

\section{MATERIALS AND METHODS}

\subsection{Treatment and experimental design}

The low energy treatment (basal diet alone) provided $88 \%$ of the energy requirements. The other treatments provided $100 \%$ of energy requirements and included the same basal diet plus either a ruminal infusion of C3 (13.4 $\mathrm{mol} / \mathrm{day}, 266 \mathrm{kcal} \mathrm{NE} / \mathrm{mol}$; [16]), or a ruminal infusion of a mixture of VFA ( $17 \mathrm{~mol} /$ day as $64 \%$ acetic acid, $21 \%$ propionic acid, and $15 \%$ butyric acid, $210 \mathrm{kcal} \mathrm{NE} / \mathrm{Lol}$; [16]), or finally a duodenal infusion of glucose $(1295 \mathrm{~g} / \mathrm{day}$, $2.75 \mathrm{Mcal} \mathrm{NE} / \mathrm{kg}$; [2]). The effect of level and type of energy was studied according to a $4 \times 4$ Latin square design over 14-day periods ( 1 week of adaptation and 1 week of measure).

\subsection{Cows and feeding}

The test was conducted in four Holstein cows (mean BW, $630 \pm 61 \mathrm{~kg}$ ) yielding $30 \pm 8$ $\mathrm{kg} / \mathrm{d}$ of milk at approximately 2 months after calving. The cows were individually fed on a restricted basal diet $(60 \%$ corn silage, $10 \%$ hay, $17.5 \%$ energy concentrate and $12.5 \%$ oil meal; DM basis) (table I) enriched with urea $(70 \mathrm{~g} / \mathrm{d})$ and sodium caseinate $(250 \mathrm{~g} / \mathrm{d})$. The diet provided $100 \%$ of the animal protein requirements [17]. All feeds were mixed and administered in two meals at 0800 and 1700 hours. The solutions were infused continuously by peristaltic pumps for $24 \mathrm{~h}$. A special device prevented direct contact between the ruminal wall and VFA. Each cow was continuously perfused with three separate solutions: VFA mixture or $\mathrm{C} 3$ and buffer (or water plus salts) in the rumen, glucose (or water) in the duodenum (table II).

\subsection{Measurements and sampling}

The amounts of feed offered and refused were weighed daily. Feed DM content was determined daily for corn silage and weekly for concentrate. At each milking, milk yield was recorded, and fat and protein contents were determined by infrared analysis (Milkoscan; Foss Electric, Hiller Ød, Denmark) for 14 separate milkings every week.

Jugular blood was sampled once each period (day 11 or 12 ) at $1 \mathrm{~h}$ before the morning meal

Table I. Ingredient and chemical composition of the basal diet and its ingredients.

\begin{tabular}{|c|c|c|c|c|c|c|c|c|}
\hline Ingredient & & $\mathrm{CP}$ & OM & Crude fiber & $\mathrm{ADF}$ & NDF & Energy & $\mathrm{PDI}^{1}$ \\
\hline & (\% of diet) & & & $(\%)$ & & & (Mcal/kg) & $(\mathrm{g} / \mathrm{kg})$ \\
\hline Corn silage & 60.0 & 7.8 & 96.5 & 17.7 & 20.6 & 37.1 & 1.56 & 60 \\
\hline Hay (ryegrass) & 10.0 & 12.2 & 91.2 & 28.8 & 34.1 & 58.8 & 1.38 & 94 \\
\hline Concentrate $^{2}$ & 17.5 & 13.5 & 90.8 & 11.8 & 15.6 & 29.6 & 1.65 & 82 \\
\hline Oil meal $^{3}$ & 12.5 & 48.7 & 93.0 & 8.2 & 10.9 & 18.7 & 1.78 & 346 \\
\hline Total diet & 100.0 & 14.3 & 94.5 & 16.6 & 19.9 & 35.7 & 1.58 & 103 \\
\hline
\end{tabular}

${ }^{1}$ Protein truly digested in the small intestine [17]; ${ }^{2}$ contains $77.4 \%$ energy concentrate ( $25 \%$ fine wheat bran, $30 \%$ sugar beet pulp, $25 \%$ barley, $10 \%$ dehydrated alfalfa, $5 \%$ molasses, $1 \%$ fat, and $4 \%$ mineral salts), $2.1 \%$ urea, $5.1 \%$ of a mixture of mineral salts and vitamins (Coopérative des Agriculteurs de Bretagne, Landerneau, France), $10.3 \% \mathrm{NaHCO}_{3}$ and $5.1 \%$ sodium caseinate; ${ }^{3}$ formaldehyde treated oil meal from soybeans $(80 \%)$ and rapeseeds $(20 \%)$. 
Table II. Nature and composition of infusions in the rumen and duodenum.

\begin{tabular}{|c|c|c|c|c|c|}
\hline \multirow[b]{2}{*}{ Site of infusion } & & \multirow[b]{2}{*}{ Basal treatment } & \multicolumn{3}{|c|}{ Infusion } \\
\hline & & & VFA & $\mathrm{C} 3^{1}$ & Glucose \\
\hline & (vol. of water) & & & & \\
\hline Rumen & $50 \mathrm{~L}$ & -2 & VFA $^{3}$ & $\mathrm{C}^{4}$ & $-^{2}$ \\
\hline Rumen & $10 \mathrm{~L}$ & salts ${ }^{5}$ & buffer ${ }^{6}$ & buffer 6 & salts ${ }^{5}$ \\
\hline Duodenum & $10 \mathrm{~L}$ & $--^{2}$ & $-^{2}$ & $-^{2}$ & glucose $^{7}$ \\
\hline
\end{tabular}

for acetate, glucose, $\beta$-hydroxybutyrate, NEFA, urea, and AA for each cow with heparinized or EDTA syringes (Sarstedt, Nümbrecht, Germany). Preparation and analysis of blood samples were the same as those described by Guinard et al. [12].

Individual samples of ruminal liquid were obtained on day 12 of each period just before the morning feeding and at 1, 2, 3,6 and $9 \mathrm{~h}$ after the morning feeding. A preservative was added to each sample, and the samples were mixed to obtain a daily pool. The VFA were determined by gas chromatography [18].

\subsection{Milk analysis - coagulation and syneresis measurements}

On day 9, $2 \mathrm{~L}$ of milk were taken from each cow at the morning milking and preserved with sodium $G$ penicillin $(2000 \mathrm{IU} / \mathrm{L})$. A subsample was reserved to determine milk fatty acid proportions by gas chromatography. Milk samples were immediately skimmed and stored at $4{ }^{\circ} \mathrm{C}$ for chemical analysis and coagulation studies. Total solids, lactose, true protein nitrogen, casein, casein fractions, soluble proteins, and total and soluble calcium were measured in the skimmed milk. After restoration of the chemical balance $\left(1 \mathrm{~h}\right.$ at $\left.35^{\circ} \mathrm{C}\right)$, rennet coagulation properties were measured with a Formagraph, either at the initial $\mathrm{pH}$ or at $\mathrm{pH} 6.5$ (standardized with lactic acid) [44]. Laboratory curd yields were also measured by centrifugation. Sampling and analyses were performed as described by Hurtaud et al. [14].

\subsection{Statistical analyses}

An ANOVA was performed for the Latin square using SAS [37]. The model included cow, period and treatment as factors of variability. The significance threshold was set at $P<0.05$ unless otherwise noted. The effect of the level of energy was tested between basal treatment and VFA mixture infusion. The effect of the type of energy was tested by comparing $\mathrm{C} 3$ or glucose infusions with VFA mixture infusion.

\section{RESULTS}

\subsection{Effect of energy level}

Regardless of the treatment, the cows ate the whole diet offered. Therefore, total $\mathrm{NE}_{\mathrm{L}}$ input was similar for the VFA mixture, C3, and glucose treatments and was lower by $12 \%$ for the basal treatment (table IV).

The VFA mixture treatment (versus the basal treatment) had no significant effect on the ruminal VFA composition (table III). Increasing the energy level by infusing VFA significantly increased milk, fat and protein yields by $0.9 \mathrm{~kg}, 42 \mathrm{~g} / \mathrm{d}$ and $37 \mathrm{~g} / \mathrm{d}$, respectively (table $I \mathrm{~V}$ ). The fat and protein contents of milk did not vary, but SNF significantly increased (table $V$ ). The casein content, the proportions of diffe- 
Table III. Effect of nature and level of source of energy on ruminal digestive paramaters.

\begin{tabular}{lccccccc}
\hline & $\begin{array}{c}\text { Basal } \\
\text { treatment }\end{array}$ & \multicolumn{3}{c}{ Infusion } & & \\
\cline { 3 - 5 } & & VFA & C3 $3^{1}$ & Glucose & RMSE $^{2}$ & $P<$ \\
\hline Concentration (mmol/L) & 82.0 & 89.7 & 84.5 & 83.1 & 4.9 & 0.225 \\
Composition (mol/100 mol) & & & & & & \\
$\quad$ Acetic & $64.5^{\mathrm{a}}$ & $63.1^{\mathrm{a}}$ & $56.6^{\mathrm{b}}$ & $63.8^{\mathrm{a}}$ & 1.5 & 0.001 \\
$\quad$ Propionic & $19.9^{\mathrm{a}}$ & $20.9^{\mathrm{a}}$ & $29.8^{\mathrm{b}}$ & $20.3^{\mathrm{a}}$ & 1.4 & 0.001 \\
$\quad$ Isobutyric & 0.8 & 0.8 & 0.9 & 0.8 & 0.1 & 0.665 \\
$\quad$ Butyric & $10.7^{\mathrm{a}}$ & $11.6^{\mathrm{a}}$ & $9.0^{\mathrm{b}}$ & $10.9^{\mathrm{a}}$ & 0.6 & 0.003 \\
$\quad$ Isovaleric & 1.9 & 1.7 & 1.9 & 2.0 & 0.2 & 0.294 \\
$\quad$ Valeric & 1.5 & 1.4 & 1.5 & 1.6 & 0.1 & 0.189 \\
Caproic & $0.6^{\mathrm{a}}$ & $0.5^{\mathrm{a}}$ & $0.3^{\mathrm{b}}$ & $0.7^{\mathrm{a}}$ & 0.1 & 0.011 \\
\hline
\end{tabular}

$\overline{\mathrm{a}, \mathrm{b}}$ Means within rows with no common superscripts differ $(P<0.05) ;{ }^{1} \mathrm{C} 3=$ propionic acid; ${ }^{2}$ root mean square error.

Table IV. Effect of level and nature of source of energy on feed intake, nutrient balance, milk yield and composition.

\begin{tabular}{|c|c|c|c|c|c|c|}
\hline & \multirow{2}{*}{$\begin{array}{l}\text { Basal } \\
\text { treatment }\end{array}$} & \multicolumn{3}{|c|}{ Infusion } & \multirow[b]{2}{*}{ RMSE $^{2}$} & \multirow[b]{2}{*}{$P<$} \\
\hline & & VFA & $\mathrm{C} 3^{1}$ & Glucose & & \\
\hline $\begin{array}{l}\mathrm{DMI}^{3}(\mathrm{~kg} / \mathrm{d}) \\
\text { Energy intake }\left(\mathrm{Mcal} \mathrm{NE}_{\mathrm{L}}\right) \\
\text { Energy balance }^{4}\left(\mathrm{Mcal} \mathrm{NE}_{\mathrm{L}}\right)\end{array}$ & $\begin{array}{l}16.6 \\
25.6^{\mathrm{b}} \\
-2.6^{\mathrm{c}}\end{array}$ & $\begin{array}{r}16.4 \\
28.9^{\mathrm{a}} \\
0.1^{\mathrm{b}}\end{array}$ & $\begin{array}{r}16.6 \\
29.0^{\mathrm{a}} \\
1.2^{\mathrm{a}}\end{array}$ & $\begin{array}{r}16.5 \\
29.0^{\mathrm{a}} \\
2.2^{\mathrm{a}}\end{array}$ & $\begin{array}{l}0.4 \\
0.7 \\
0.6\end{array}$ & $\begin{array}{l}0.981 \\
0.001 \\
0.001\end{array}$ \\
\hline $\begin{array}{l}\text { CP intake }(\mathrm{g}) \\
\text { Protein intake }\left(\mathrm{g} \mathrm{PDI}^{5}\right)\end{array}$ & $\begin{array}{l}2799 \\
1690\end{array}$ & $\begin{array}{l}2787 \\
1681\end{array}$ & $\begin{array}{l}2788 \\
1682\end{array}$ & $\begin{array}{l}2778 \\
1674\end{array}$ & $\begin{array}{l}35 \\
27\end{array}$ & $\begin{array}{l}0.870 \\
0.876\end{array}$ \\
\hline Protein balance ${ }^{3}\left(\mathrm{~g} \mathrm{PDI}^{5}\right)$ & 87 & 20 & -20 & 12 & 42 & 0.056 \\
\hline $\begin{array}{l}\text { Milk }(\mathrm{kg} / \mathrm{d}) \\
4 \% \text { FCM }(\mathrm{kg} / \mathrm{d}) \\
\text { Fat }\end{array}$ & $\begin{array}{l}26.7^{b} \\
24.7^{b}\end{array}$ & $\begin{array}{l}27.6^{\mathrm{a}} \\
25.7^{\mathrm{a}}\end{array}$ & $\begin{array}{l}28.1^{a} \\
24.3^{b}\end{array}$ & $\begin{array}{l}26.3^{b} \\
22.8^{c}\end{array}$ & $\begin{array}{l}0.4 \\
0.4\end{array}$ & $\begin{array}{l}0.003 \\
0.001\end{array}$ \\
\hline $\begin{array}{l}\text { Fat } \\
\qquad \mathrm{g} / \mathrm{d} \\
\mathrm{g} / \mathrm{kg}\end{array}$ & $\begin{array}{l}936^{\mathrm{b}} \\
35.8^{\mathrm{a}}\end{array}$ & $\begin{array}{l}978^{\mathrm{a}} \\
36.1^{\mathrm{a}}\end{array}$ & $\begin{array}{l}867^{\mathrm{c}} \\
31.5^{\mathrm{b}}\end{array}$ & $\begin{array}{l}818^{\mathrm{d}} \\
31.8^{\mathrm{b}}\end{array}$ & $\begin{array}{l}23 \\
1.0\end{array}$ & $\begin{array}{l}0.001 \\
0.001\end{array}$ \\
\hline $\begin{array}{l}\text { True protein } \\
\text { g/d } \\
\mathrm{g} / \mathrm{kg}\end{array}$ & $\begin{array}{l}762^{\mathrm{c}} \\
28.9^{\mathrm{b}}\end{array}$ & $\begin{array}{l}799^{b} \\
29.2^{b}\end{array}$ & $\begin{array}{l}825^{\mathrm{a}} \\
29.6^{\mathrm{ab}}\end{array}$ & $\begin{array}{l}799^{\mathrm{b}} \\
30.7^{\mathrm{a}}\end{array}$ & $\begin{array}{c}14 \\
0.7\end{array}$ & $\begin{array}{l}0.004 \\
0.032\end{array}$ \\
\hline
\end{tabular}

${ }^{1} \mathrm{C} 3$ = propionic acid; ${ }^{2}$ root mean square error; ${ }^{3}$ without infusion; all the other results are with infusions; ${ }^{4}$ difference between intake and requirement [17]; ${ }^{5}$ protein digested in the small intestine [17].

$\mathrm{a}, \mathrm{b}, \mathrm{c}, \mathrm{d}$ Means within rows with no common superscripts differ $(P<0.05)$.

rent types of casein ( $\alpha-, \beta$ - and $\kappa$-casein) and the $\mathrm{Ca}$ content and distribution (colloidal and soluble $\mathrm{Ca}$ ) of the skimmed milk were unchanged (table $V$ ). The proportion of fatty acids changed with the increase in energy level. The proportions of $\mathrm{C}_{18: 1}$, and $\mathrm{C}_{18: 0}$ significantly decreased, whereas that of $\mathrm{C}_{16: 0}$ significantly increased (table VI).

Plasma acetate, glucose, and $\beta$-hydroxybutyrate contents (table VII) were slightly higher for cows infused with VFA mix- 
Table V. Effect of level and nature of source of energy on skim milk composition.

\begin{tabular}{|c|c|c|c|c|c|c|}
\hline & \multirow{2}{*}{$\begin{array}{c}\text { Basal } \\
\text { treatment }\end{array}$} & \multicolumn{3}{|c|}{ Infusion } & \multirow[b]{2}{*}{$\mathrm{RMSE}^{2}$} & \multirow[b]{2}{*}{$P<$} \\
\hline & & VFA & $\mathrm{C} 3^{1}$ & Glucose & & \\
\hline Casein $(\mathrm{g} / \mathrm{kg})$ & $23.4^{\mathrm{b}}$ & $23.6^{\mathrm{ab}}$ & $24.4^{\mathrm{ab}}$ & $25.4^{\mathrm{a}}$ & 0.7 & 0.024 \\
\hline Soluble proteins $(\mathrm{g} / \mathrm{kg})$ & $5.5^{\mathrm{b}}$ & $5.6^{\mathrm{b}}$ & $5.8^{\mathrm{ab}}$ & $6.1^{\mathfrak{a}}$ & 0.2 & 0.038 \\
\hline NPN $(g / k g)$ & 0.21 & 0.20 & 0.20 & 0.20 & 0.08 & 0.448 \\
\hline Colloidal $\mathrm{Ca}(\mathrm{mg} / \mathrm{kg})$ & 823 & 779 & 831 & 855 & 32 & 0.068 \\
\hline Lactose $(\mathrm{g} / \mathrm{kg})$ & 45.5 & 47.3 & 48.1 & 45.1 & 3.6 & 0.635 \\
\hline
\end{tabular}

a, b Within rows, means with no common superscripts $\operatorname{differ}(P<0.05) ;{ }^{1} \mathrm{C} 3=$ propionic acid; ${ }^{2}$ root mean square error.

Table VI. Effect of level and nature of source of energy on milk fatty acids proportion.

\begin{tabular}{|c|c|c|c|c|c|c|}
\hline & \multirow{2}{*}{$\begin{array}{c}\text { Basal } \\
\text { treatment }\end{array}$} & \multicolumn{3}{|c|}{ Infusion } & \multirow[b]{2}{*}{$\mathrm{RMSE}^{2}$} & \multirow[b]{2}{*}{$P<$} \\
\hline & & VFA & $\mathrm{C} 3^{\mathrm{I}}$ & Glucose & & \\
\hline \multicolumn{7}{|l|}{ Fatty acids (\%) } \\
\hline $\mathrm{C}_{4: 0}$ & $2.52^{\mathrm{a}}$ & $2.43^{\mathrm{ab}}$ & $2.08^{b}$ & $2.20^{\mathrm{ab}}$ & 0.15 & 0.023 \\
\hline $\mathrm{C}_{6: 0}$ & $1.83^{\mathrm{a}}$ & $1.80^{\mathrm{a}}$ & $1.57^{\mathrm{b}}$ & $1.77^{\mathrm{a}}$ & 0.07 & 0.005 \\
\hline $\mathrm{C}_{8: 0}$ & $1.21^{b}$ & $1.17^{\mathrm{b}}$ & $1.06^{\mathrm{c}}$ & $1.26^{\mathrm{a}}$ & 0.04 & 0.002 \\
\hline $\mathrm{C}_{10: 0}$ & $3.14^{\mathrm{b}}$ & $3.20^{b}$ & $3.30^{\mathrm{b}}$ & $3.83^{\mathrm{a}}$ & 0.15 & 0.002 \\
\hline $\mathrm{C}_{12: 0}$ & $3.97^{\mathrm{b}}$ & $4.14^{\mathrm{b}}$ & $4.18^{\mathrm{b}}$ & $5.28^{\mathrm{a}}$ & 0.18 & 0.001 \\
\hline$C_{14: 1}$ & $1.47^{\mathrm{b}}$ & $1.35^{\mathrm{b}}$ & $1.34^{\mathrm{b}}$ & $1.84^{\mathrm{a}}$ & 0.20 & 0.037 \\
\hline $\mathrm{C}_{14: 0}$ & $13.0^{\mathrm{bc}}$ & $13.6^{\mathrm{b}}$ & $12.8^{\mathrm{c}}$ & $14.4^{\mathrm{a}}$ & 0.41 & 0.005 \\
\hline iso- $\mathrm{C}_{15}$ & 0.23 & 0.22 & 0.20 & 0.19 & 0.05 & 0.602 \\
\hline$C_{15: 1}$ & 0.54 & 0.48 & 0.48 & 0.46 & 0.10 & 0.721 \\
\hline$C_{15: 0}$ & $1.27^{\mathrm{b}}$ & $1.32^{\mathrm{b}}$ & $2.22^{\mathrm{a}}$ & $1.55^{\mathrm{b}}$ & 0.18 & 0.001 \\
\hline$C_{16: 1}$ & 1.78 & 1.88 & 1.89 & 2.04 & 0.16 & 0.266 \\
\hline$C_{16: 0}$ & $33.9^{\mathrm{c}}$ & $37.7^{\mathrm{ab}}$ & $36.3^{b}$ & $38.5^{\mathrm{a}}$ & 0.91 & 0.002 \\
\hline iso- $\mathrm{C}_{17: 0}$ & 0.31 & 0.28 & 0.28 & 0.22 & 0.06 & 0.318 \\
\hline$C_{17: 1}$ & 0.81 & 0.70 & 0.77 & 0.66 & 0.09 & 0.169 \\
\hline $\mathrm{C}_{17: 0}$ & 0.53 & 0.57 & 0.76 & 0.61 & 0.10 & 0.060 \\
\hline $\mathrm{C}_{18: 2}$ & 2.37 & 2.18 & 2.19 & 2.14 & 0.16 & 0.273 \\
\hline $\mathrm{C}_{18: 1}$ & $20.2^{\mathrm{a}}$ & $17.7^{b}$ & $19.1^{\mathrm{a}}$ & $15.7^{\mathrm{c}}$ & 0.65 & 0.001 \\
\hline $\mathrm{C}_{18: 0}$ & $10.9^{\mathrm{a}}$ & $9.3^{\mathrm{b}}$ & $9.5^{\mathrm{b}}$ & $7.3^{c}$ & 0.58 & 0.001 \\
\hline \multicolumn{7}{|l|}{ Fatty acids (g/d) } \\
\hline $\mathrm{C}_{4: 0}$ to $\mathrm{C}_{12: 0}$ & $102^{b}$ & $107^{a}$ & $91^{\mathrm{c}}$ & $101^{\mathrm{b}}$ & 2.9 & 0.001 \\
\hline$C_{14: 1}+C_{14: 0}$ & $116^{b}$ & $125^{\mathrm{a}}$ & $105^{c}$ & $114^{b}$ & 3.3 & 0.001 \\
\hline $\mathrm{C}_{16: 1}+\mathrm{C}_{16: 0}$ & $287^{\mathrm{b}}$ & $333^{\mathrm{a}}$ & $285^{\mathrm{b}}$ & $286^{\mathrm{b}}$ & 9.8 & 0.001 \\
\hline $\mathrm{C}_{18: 2}+\mathrm{C}_{18: 1}+\mathrm{C}_{18: 0}$ & $269^{\mathrm{a}}$ & $245^{\mathrm{b}}$ & $239^{\mathrm{b}}$ & $176^{\mathrm{c}}$ & 13.7 & 0.001 \\
\hline
\end{tabular}


Table VII. Effect of level and nature of source of energy on blood parameters.

\begin{tabular}{|c|c|c|c|c|c|c|}
\hline & \multirow{2}{*}{$\begin{array}{c}\text { Basal } \\
\text { treatment }\end{array}$} & \multicolumn{3}{|c|}{ Infusion } & \multirow[b]{2}{*}{$\mathrm{RMSE}^{2}$} & \multirow[b]{2}{*}{$P<$} \\
\hline & & VFA & C $3^{1}$ & Glucose & & \\
\hline Glucose $(\mathrm{mg} / 100 \mathrm{~mL})$ & 69.9 & 74.2 & 73.4 & 72.5 & 3.0 & 0.281 \\
\hline NEFA $(\mu \mathrm{mol} / \mathrm{L})$ & 138 & 85 & 94 & 82 & 58 & 0.532 \\
\hline Acetate $(\mathrm{mg} / 100 \mathrm{~mL})$ & $3.8^{\mathrm{b}}$ & $4.4^{\mathrm{a}}$ & $3.0^{\mathrm{c}}$ & $2.9^{c}$ & 0.2 & 0.001 \\
\hline$\beta$-hydroxybutyrate (mg & $\mathrm{mL}) 3.9^{\mathrm{a}}$ & $4.3^{\mathrm{a}}$ & $2.4^{\mathrm{b}}$ & $2.7^{\mathrm{b}}$ & 0.3 & 0.001 \\
\hline Urea $(\mathrm{mg} / 100 \mathrm{~mL})$ & 22.2 & 20.8 & 20.6 & 18.8 & 2.9 & 0.467 \\
\hline
\end{tabular}

a,b,c Within rows, means with no common superscripts differ $(P<0.05) ;{ }^{1} \mathrm{C} 3=$ propionic acid; ${ }^{2}$ root mean square error.

ture, but only the increase in acetate was significant. Urea and total AA contents were unaffected, but the Glu and Gln concentrations were significantly increased (table VIII).

\subsection{Effect of energy type}

For cows receiving infusions of VFA mixture, C3 or glucose, the amount of consumed diet was the same. So the differences registered between the three treatments were only imputed to the nature of the energy infused.

As expected, the $\mathrm{C} 3$ treatment (versus the VFA mixture treatment) significantly increased ruminal C3 $(29.8 \%$ versus $20.9 \%)$ at the expense of acetic acid (56.6\% versus $63.1 \%)$, butyric acid $(9.0 \%$ versus $11.6 \%)$, and caproic acid $(0.3 \%$ versus $0.5 \%)$. The glucose treatment did not affect the VFA profile (table III).

Relative to the VFA mixture treatment, the C3 treatment significantly increased the protein yield $(26 \mathrm{~g} / \mathrm{d})$, although both milk yield and protein content were scarcely changed. In contrast, the glucose treatment did not alter protein yield, because milk yield decreased $(1.3 \mathrm{~kg} / \mathrm{d})$, and protein content increased $(1.5 \mathrm{~g} / \mathrm{kg})$, both significantly. Proportions of different types of casein $(\alpha-, \beta$ - and $\kappa$-casein) did not change for cows infused with either C3 or glucose. Soluble proteins significantly increased for cows infused with glucose $(0.5 \mathrm{~g} / \mathrm{kg})$. Colloidal calcium tended to increase for cows infused with either treatment (table $V)$. Both $\mathrm{C} 3$ and glucose treatments significantly decreased the fat content (close to $4.5 \mathrm{~g} / \mathrm{kg}$ ) and yield (111 g, C3 treatment; $160 \mathrm{~g}$, glucose treatment) (table IV).

Although fat content was similarly decreased, the secretion of individual fatty acids ( $g$ per d) was affected differently by the C3 or glucose treatments, except for $\mathrm{C}_{4: 0}$, which was largely decreased for cows infused with either treatment (figure $I$ and table $V I)$. For the even-numbered fatty acids, the $\mathrm{C} 3$ treatment (versus VFA treatment) depressed the secretion of $\mathrm{C}_{6: 0}, \mathrm{C}_{8: 0}$, $\mathrm{C}_{14}$, and $\mathrm{C}_{16}(\mathrm{~g} / \mathrm{d})$. Conversely, with the glucose treatment (versus VFA treatment), the large decrease observed in $\mathrm{C}_{4: 0}$ secretion was gradually counterbalanced by increases of other short and medium fatty acids. Consequently, the differences between VFA mixture and glucose infusions decreased as the chains of fatty acids increased and even for $C_{12: 0}$, glucose infusion induced an increase compared to VFA infusion. A reverse trend occurred for $\mathrm{C}_{18}$ so that its secretion was largely depressed in contrast to what occurred following the C3 infusion, which had no effect on $\mathrm{C}_{18}$ yield (figure 1). The odd-numbered fatty acids (mainly $\mathrm{C}_{15}$ ) were significantly increased following the $\mathrm{C} 3$ treatment but not for the glucose treatment. 
Table VIII. Effect of level and nature of source of energy on blood plasma AA.

\begin{tabular}{|c|c|c|c|c|c|c|}
\hline & \multirow{2}{*}{$\begin{array}{c}\text { Basal } \\
\text { treatment }\end{array}$} & \multicolumn{3}{|c|}{ Infusion } & \multirow[b]{2}{*}{$\mathrm{RMSE}^{2}$} & \multirow[b]{2}{*}{$P<$} \\
\hline & & VFA & $C 3^{1}$ & Glucose & & \\
\hline \multicolumn{7}{|c|}{$\operatorname{EAA}^{3}(\mathrm{mg} / 100 \mathrm{~mL})$} \\
\hline Lys & 1.37 & 1.26 & 1.04 & 1.09 & 0.27 & 0.386 \\
\hline His & 0.85 & 0.88 & 0.82 & 0.84 & 0.11 & 0.886 \\
\hline Arg & 1.65 & 1.49 & 1.28 & 1.36 & 0.29 & 0.369 \\
\hline Thr & 1.14 & 1.21 & 1.15 & 1.20 & 0.20 & 0.949 \\
\hline Val & 3.01 & 2.75 & 2.19 & 2.13 & 0.41 & 0.057 \\
\hline Met & 0.31 & 0.31 & 0.31 & 0.31 & 0.05 & 0.998 \\
\hline Ile & 1.42 & 1.39 & 1.03 & 1.03 & 0.20 & 0.058 \\
\hline Leu & 1.71 & 1.58 & 1.16 & 1.18 & 0.28 & 0.076 \\
\hline Phe & 0.90 & 0.92 & 0.79 & 0.85 & 0.14 & 0.588 \\
\hline Total & 12.4 & 11.8 & 9.8 & 10.0 & 1.82 & 0.216 \\
\hline \multicolumn{7}{|c|}{$\mathrm{NEAA}^{4}(\mathrm{mg} / 100 \mathrm{~mL})$} \\
\hline Asp & 0.06 & 0.06 & 0.10 & 0.05 & 0.06 & 0.695 \\
\hline Asn & 0.75 & 0.83 & 0.75 & 0.83 & 0.12 & 0.724 \\
\hline Ser & 1.31 & 1.25 & 1.17 & 1.28 & 0.20 & 0.793 \\
\hline Glu & $0.49^{\mathrm{b}}$ & $0.61^{\mathrm{a}}$ & $0.51^{\mathrm{b}}$ & $0.51^{b}$ & 0.04 & 0.026 \\
\hline Gln & $2.16^{\mathrm{b}}$ & $2.64^{\mathrm{a}}$ & $2.84^{\mathrm{a}}$ & $2.73^{\mathrm{a}}$ & 0.24 & 0.027 \\
\hline Gly & 3.66 & 3.51 & 3.31 & 4.17 & 0.58 & 0.287 \\
\hline Ala & 2.20 & 2.29 & 1.88 & 1.84 & 0.28 & 0.149 \\
\hline Tyr & 0.71 & 0.71 & 0.65 & 0.72 & 0.14 & 0.869 \\
\hline Cys & 0.61 & 0.60 & 0.65 & 0.62 & 0.08 & 0.867 \\
\hline Tau & 0.59 & 0.43 & 0.51 & 0.43 & 0.13 & 0.434 \\
\hline Orn & 0.85 & 0.74 & 0.65 & 0.74 & 0.14 & 0.345 \\
\hline Cit & 1.43 & 1.16 & 1.18 & 1.26 & 0.21 & 0.348 \\
\hline Total & 12.7 & 12.2 & 11.4 & 12.4 & 1.64 & 0.704 \\
\hline Total AA & 25.0 & 24.0 & 21.1 & 22.4 & 3.37 & 0.443 \\
\hline
\end{tabular}

a,b Within rows, means with no common superscripts differ $(P<0.05) ;{ }^{1} \mathrm{C} 3=$ propionic acid; ${ }^{2}$ root mean square error; ${ }^{3}$ essential amino acids; ${ }^{4}$ non-essential amino acids.

The $\mathrm{C} 3$ and glucose treatments had the same effects on energy and plasma nitrogen metabolites. Relative to the VFA mixture treatment, $\mathrm{C} 3$ and glucose treatments did not affect the NEFA and glucose contents, but both significantly decreased the $\beta$-hydroxybutyrate and acetate contents (table VII). The C3 and glucose treatments had no effect on plasma urea and tended to decrease total essential AA in plasma. Contents of branched AA tended to decrease for cows infused with either $\mathrm{C} 3$ or glucose (respectively, 1.3 and 1.4 $\mathrm{mg} / 100 \mathrm{~mL} ; P<0.063$ ). Among the nonessential AA, the concentration of Glu decreased, and that of Ala tended to decrease $(P<0.149)$ (table VIII).

\subsection{Milk coagulation properties}

The increase in energy had no effect on milk coagulation properties, except on curd firmness, which significantly increased at standardized $\mathrm{pH}$ with the VFA mixture treatment (table IX).

The source of energy had no effect on curd yields. At the initial $\mathrm{pH}$, the $\mathrm{C} 3$ treatment did not change milk coagulation properties, but the glucose treatment signifi- 


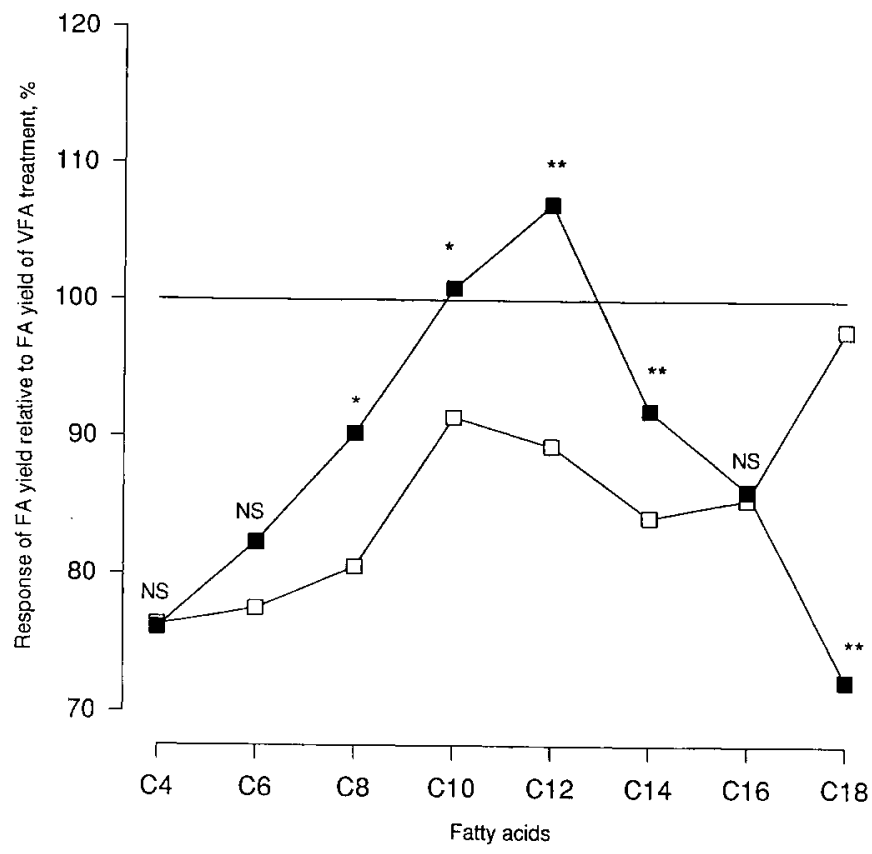

Figure 1. Responses in the individual fatty acid yields of milk to $\mathrm{C} 3(\square)$ or glucose $(\boldsymbol{\square})$ infusions as compared to VFA infusions (** $: P<0.01{ }^{*}: P<0.05$; NS : $P>0.05$ ).

Table IX. Effect of level and nature of source of energy on milk coagulation properties.

\begin{tabular}{|c|c|c|c|c|c|c|}
\hline & \multirow{2}{*}{$\begin{array}{c}\text { Basal } \\
\text { treatment }\end{array}$} & \multicolumn{3}{|c|}{ Infusion } & \multirow[b]{2}{*}{ RMSE $^{2}$} & \multirow[b]{2}{*}{$P<$} \\
\hline & & VFA & $\mathrm{C} 3^{1}$ & Glucose & & \\
\hline \multicolumn{7}{|l|}{ Curd yield (\%) } \\
\hline Fresh & 13.3 & 13.7 & 14.3 & 14.9 & 1.2 & 0.328 \\
\hline $\mathrm{N}$ & 76.5 & 76.8 & 77.2 & 77.5 & 0.7 & 0.578 \\
\hline Dry & 39.2 & 39.1 & 40.6 & 40.7 & 2.1 & 0.303 \\
\hline \multicolumn{7}{|l|}{ Coagulating properties } \\
\hline At initial $\mathrm{pH}$ & 6.65 & 6.67 & 6.66 & 6.63 & 0.02 & 0.117 \\
\hline Rennet clotting time, $\min$ & $15.0^{\mathrm{a}}$ & $14.8^{\mathrm{a}}$ & $14.7^{\mathrm{a}}$ & $13.2^{\mathrm{b}}$ & 0.5 & 0.009 \\
\hline Rate of firming, min & $7.5^{\mathrm{a}}$ & $7.2^{\mathrm{a}}$ & $6.7^{\mathrm{a}}$ & $5.2^{\mathrm{b}}$ & 0.6 & 0.006 \\
\hline Curd firmness, $\mathrm{mm}$ & $33.4^{b}$ & $36.5^{\mathrm{ab}}$ & $37.9^{\mathrm{ab}}$ & $43.5^{a}$ & 3.6 & 0.036 \\
\hline \multicolumn{7}{|l|}{ At standardized $\mathrm{pH}^{3}$} \\
\hline Rennet clotting time, $\min$ & 8.7 & 8.4 & 7.6 & 8.5 & 1.1 & 0.505 \\
\hline Rate of firming, min & 4.3 & 4.1 & 3.6 & 3.3 & 0.5 & 0.070 \\
\hline Curd firmness, $\mathrm{mm}$ & $47.7^{\mathrm{c}}$ & $50.4^{b}$ & $53.6^{\mathrm{a}}$ & $55.1^{\mathrm{a}}$ & 1.8 & 0.004 \\
\hline
\end{tabular}

a,b,c Within rows, means with no common superscripts $\operatorname{differ}(P<0.05) ;{ }^{1} \mathrm{C} 3=$ propionic acid; $^{2}$ root mean square error; ${ }^{3} \mathrm{pH}=6.5$. 
cantly decreased rennet clotting time and firming rate. At the standardized $\mathrm{pH}$, both the $\mathrm{C} 3$ and glucose treatments increased curd firmness (table IX).

\section{DISCUSSION}

The VFA pattern and glucose availability were significantly modified by the C3 and glucose infusions, although these changes were not unusual when compared with some types of diets, either with high levels of concentrate [4] or with rapidly degradable starchy concentrates [30].

Under our experimental conditions, the ruminal VFA profiles observed did not indicate any modification in the fermentation products of the feed portion when extra VFA were infused. Similar observations have been made previously [35], not only with regards to VFA but also for ruminal liquid turnover and duodenal $A A$ flow. It can also be reasonably assumed that the absorption of the extra VFA was complete. The absorption of the infused glucose should also have been nearly complete. No disturbance was observed regarding the glucose absorption, and a 73-90\% absorption rate has been previously found with similar doses of glucose $[22,23]$. Therefore, it can be reasonably assumed that glucogenic nutrients were increased by $\mathrm{C} 3$ or glucose infusions.

\subsection{Protein synthesis}

Increasing the energy level (from the basal treatment to VFA mixture treatment) or modifying ruminal fermentation products with the same total energy supply (from VFA mixture treatment to C3 treatment) had an almost identical effect on the milk protein synthesis ( $37 \mathrm{~g}$ for cows administered the VFA mixture treatment and $26 \mathrm{~g}$ for cows infused with C3). These slight increases in protein synthesis appea- red to have different causes. The increase in energy level was reflected by an increase in milk yield and a small increase in protein content $(0.1 \mathrm{~g} / \mathrm{kg}$ for each extra Mcal of energy). That increase in protein content, induced by the higher energy level, was slightly less than that induced by concentrates $(0.15$ to $0.3 \mathrm{~g} / \mathrm{kg}$ for each extra Mcal of $\left.\mathrm{NE}_{\mathrm{L}}\right)[9,32,39]$ In our trial, when changing from the basal treatment to VFA mixture infusion, only the energy level was altered, without any effect on AA supply or on microbial synthesis. The increase in protein content could only be linked to a higher ATP availability for milk protein synthesis. This contrasts with the effects of energy concentrates that modify ATP and AA together. Moreover, our treatment periods were relatively short (14 days) compared to trials on energy concentrates (often at least 4 weeks) that could also explain the small effect of energy level on milk synthesis.

The slight increase $(26 \mathrm{~g} / \mathrm{d})$ in protein synthesis recorded for the cows infused with $\mathrm{C} 3$, relative to that for cows infused with VFA mixture, was in the same range than previously recorded responses (from -56 to $72 \mathrm{~g} / \mathrm{d}$; table $X$ gathering trials where intake was not dramatically decreased by energy treatments). In view of the results of table $X$, protein synthesis appeared not to be improved by increasing ruminal C3 level in isoenergetic situations (mean value, $-30 \mathrm{~g} / \mathrm{d}$ ) ([5] (trial 1); [13, 14] and the result of this trial). The increased energy level due to $\mathrm{C} 3$ infusions seemed to have a slight positive effect on protein synthesis (mean value $34 \mathrm{~g} / \mathrm{d}$ ) ([5] (trial 2); [33, 34, 42]. This increase (8.3 g of protein/ Mcal $\mathrm{NE}_{\mathrm{L}}$ added) is even lower than that obtained by increasing energy level with the VFA mixture in this trial (11.2 $\mathrm{g}$ of protein/ Mcal $\mathrm{NE}_{\mathrm{L}}$ added).

The response of milk protein yield $(0 \mathrm{~g} / \mathrm{d})$ observed in this trial with glucose infusion is rather lower than responses generally observed in identical energetic 


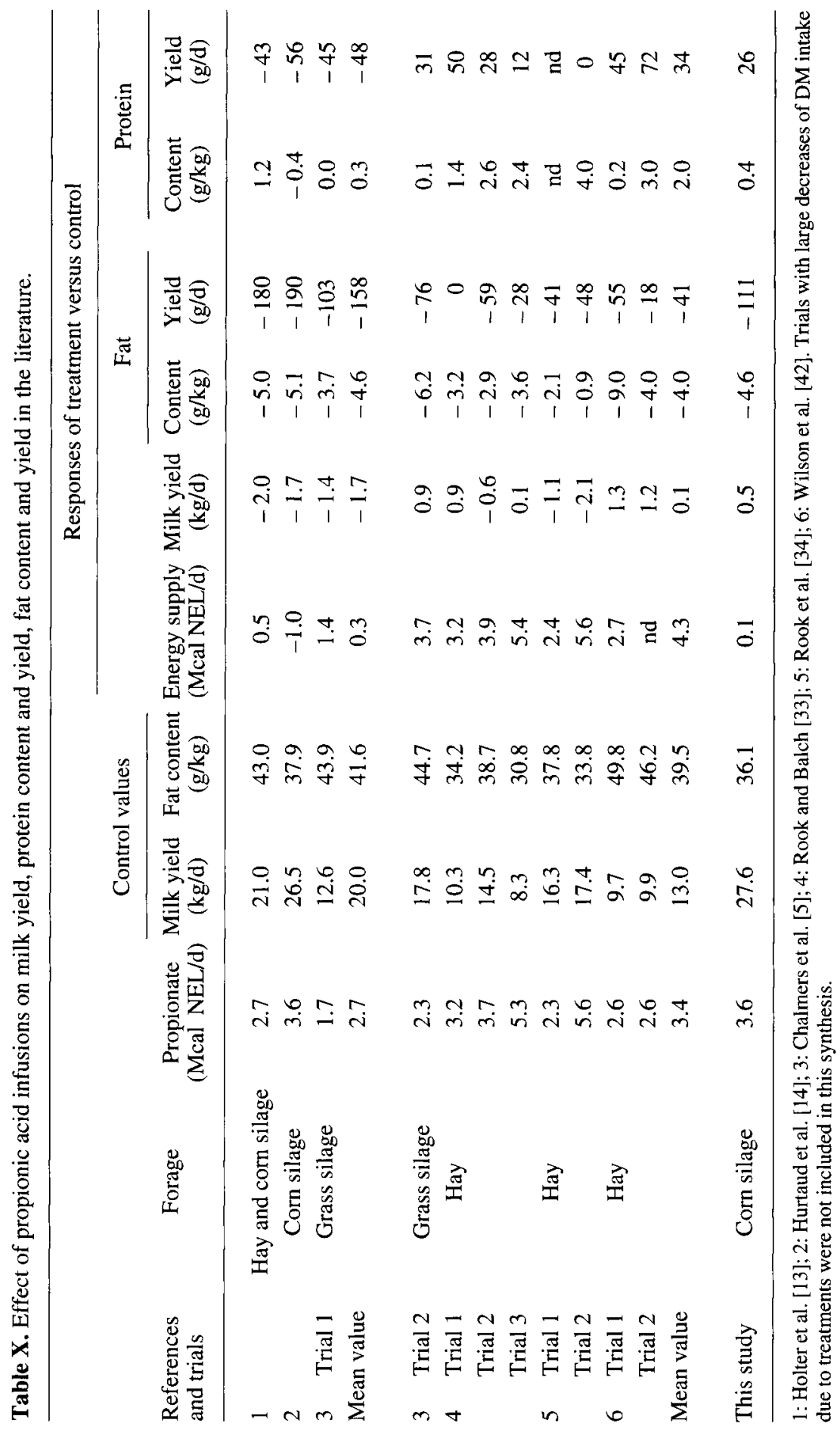


situations (mean value, $18 \mathrm{~g} / \mathrm{d}$; table $X I$ ) except for Clark et al. [6] ( $-20 \mathrm{~g} / \mathrm{d})$. However, in high energetic situations [27, 43], protein yield decreased with glucose infusions. This decrease could be related to the negative effect on milk yield that seemed to be observed when glucose is infused with a corn based diet $[24,27]$ or with diets rich in concentrates $[29,43]$. This trial does not bring any support to the hypothesis that increasing $\mathrm{C} 3$ or glucose supply could spare glucogenic AA and hence increase milk protein content. Indeed, all the infusions (VFA mixture, C3 and glucose) provided the same amounts of energy and PDI and they did not increase the level of glucogenic AA in plasma comparatively to basal treatment. In contrast, the amount of branched AA decreased as for Clark et al. [6], Whitelaw et al. [43] and Dhiman et al. [8] with glucose infusions. These AA were perhaps used preferentially for muscle synthesis [3] under hormonal control.

The infusion of $\mathrm{C} 3$ or glucose was presumably the cause of increased rate of glucose supply as for Judson and Leng [20] and Seal and Parker [38] with C3 infusion and as for Clark et al. [6] and Amaral et al. [1] for glucose infusion. The difference between these two energy nutrients could be linked to the very nature of each one and to their infusion sites (rumen or intestine). Indeed, by comparing intravenous infusions of glucose and C3, Fisher and Elliot [10] found no significant and no different effect of these nutrients on milk protein content. However, this lack of difference in the intravenous comparison could be related to the same infusion sites and to short periods of infusion.

\subsection{Fat synthesis}

Decreasing the energy level, switching from acetic to propionic fermentation and increasing the duodenal glucose flux induced a decrease in fat synthesis.
Under the basal treatment, cows were energy deficient and mobilized much body fat. The milk fat became rich in long-chain fatty acids at the expense of the short- and medium-chain fatty acids in response to the variations of their respective plasma precursors (acetate, $\beta$-hydroxybutyrate, NEFA). These modifications agree with that classically observed when cows are underfed [19].

Infusion of $\mathrm{C} 3$ or glucose induced similar decreases in fat content $(-4.5 \mathrm{~g} / \mathrm{kg})$, but the decrease of fat yield, as in Fisher and Elliot [10], was more important for cows infused with glucose $(-160 \mathrm{~g}$ versus $-111 \mathrm{~g}$ for $\mathrm{C} 3$ infusion) because of the concomitant decrease in milk yield in our trial. Such decreases are similar to the published data for fat content but much greater for fat yield as summarized for $\mathrm{C} 3$ infusions (table $X$ ) and for glucose infusions (table XI) ([5] (trial 2); [6, 11, 24, $27,29,33,34,41-43]$ ) except in isoenergetic situations with C3 infusions [5] (trial 2); [13, 14] (table X).

With glucose infusion, as opposed to VFA mixture infusion, and keeping in mind the unchanged supply of feed and microbial lipids, the differences in milk fatty acids patterns appear to indicate that: 1) the net contribution of body lipids to milk fat synthesis was greatly depressed perhaps because of lower lipolysis or greater lipogenesis [21] as indicated by a decrease in $\mathrm{C}_{18} ; 2$ ) de novo synthesis was also depressed, but to a much lesser extent; 3 ) the elongation process was much more active as shown by the increase in intermediate chain length fatty acids $\left(\mathrm{C}_{12: 0}\right)$ and the concomitant large decrease in the very short-chain fatty acids. This could also result partly from the lowered availability of precursors (acetate and $\beta$-hydroxybutyrate).The increased activity of the elongation process could also have affected positively the synthesis of $\mathrm{C}_{14}$ and $\mathrm{C}_{16}$. However, because of their dual origin [26], and of the postulated decrease 


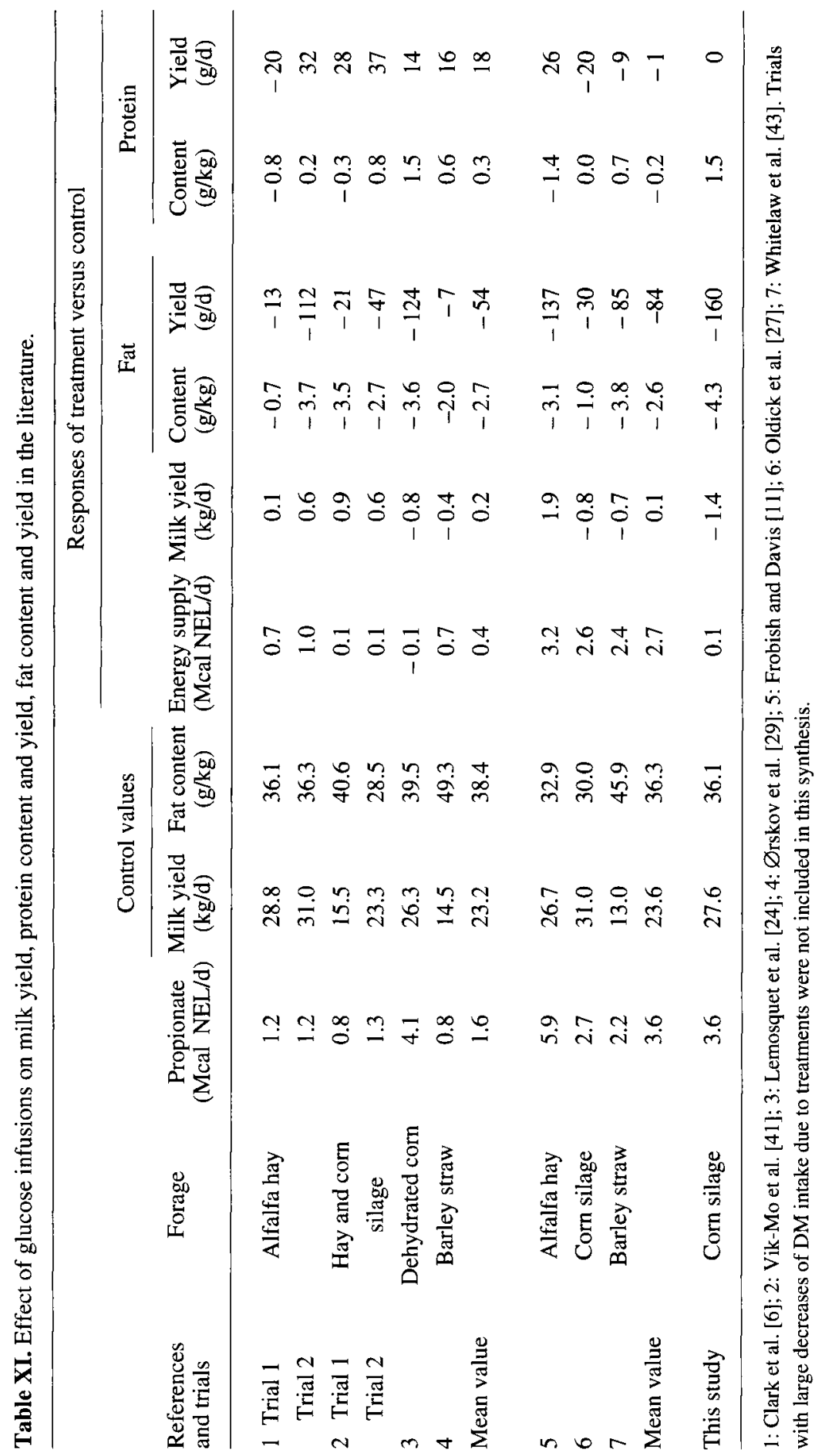


of adipose tissue lipolysis or increase of lipogenesis, such an effect would be masked. With the $\mathrm{C} 3$ infusion, those changes appear to be intermediate. In particular, the net contribution of body lipids to milk fat was less affected than it had been with glucose infusion. Some of the energy supplied in the form of C3 might not have participated in hepatic neoglucogenesis and so would have been a limiting factor in short-chain fatty acid synthesis in the udder because of the reduction of energy under the form of glucose. Conversely, circulating $\mathrm{C} 3$ not captured in the liver might have been utilized in priority by the udder for synthesizing odd-numbered fatty acids, $C_{15}$ in particular [14], because $C_{17}$ has a dual origin [25]. Results from Hurtaud et al. [14] and Lemosquet et al. [24] give similar support to the above possibilities.

\subsection{Milk coagulation properties}

Level and nature of energy did not have a major effect on milk coagulation properties. At the initial $\mathrm{pH}$, the significant effect of glucose infusion was only due to a slight decrease in milk pH [31]. At a standardized $\mathrm{pH}$, the improvement in curd firmness with the change of nature of energy (C3 or glucose versus VFA mixture infusion) seemed to be a consequence of the increase in protein, casein and colloidal calcium [15, 31].

\section{CONCLUSION}

Changes in milk protein secretion or protein content with altered energy nutrient patterns in isoenergetic situations were not very large, nor consistent as they differed between C3 and glucose treatments. No support was found for the hypothesis that sparing glucogenic AA by providing glucogenic nutrients to the liver would result in an increased protein secretion.
Both glucogenic nutrients had a similar adverse effect on fat contents but acted through different metabolic pathways. It was postulated that adipose tissue lipolysis or lipogenesis but also fatty acid elongation differed according to the energy nutrient patterns. Further information about hormonal control is necessary to complete the picture.

Concentrate manipulation (more concentrate or improved forage intake) increasing the energy supply, seems to be a better way to increase protein secretion. Experimentally, these conclusions seemed to be true for corn based diets with medium levels of concentrates which provided significant amounts of glucogenic nutrients.

Further work is required with other diets differing in starch content and degradability (as for instance grass silage). In order to manage the fat yield that is used at the basis of milk quota determination, it could also be of interest to explore the relationships between doses of glucogenic nutrients (as C3 or glucose) and secretion of fat in the milk of dairy cows.

\section{ACKNOWLEDGEMENTS}

The authors gratefully thank the late $\mathrm{H}$. Hétault and his crew for taking care of the cows and I. Jicquel, L. Quintard, S. Rigault, L. Toullec and M. Vérité for the laboratory analyses. Our thanks also to Philip Rousseau-Cunningham for the English translation.

\section{REFERENCES}

[1] Amaral. D.M., Veenhuizen J.J., Drackley J.K., Cooley M.H., McGilliard A.D., Young J.W., Metabolism of propionate, glucose, and carbon dioxide as affected by exogenous glucose in dairy cows at energy equilibrium, $\mathrm{J}$. Dairy Sci. 73 (1990) 1244-1254.

[2] Armstrong D.G., Blaxter K.L., The utilization of the energy of carbohydrate by ruminants, in: Proc. 2nd Symp. on Energy Metabolism, EAAP publ. No 10, the Netherlands, Wageningen, 1961, pp. 187-199. 
[3] Brockman R.P., Laarveld B., Hormonal regulation of metabolism in ruminants, Livest. Prod. Sci. 14 (1986) 313-334.

[4] Casper D.P., Schingoethe D.J., Eisenbeisz W.A., Response of early lactation dairy cows fed diets varying in source of nonstructural carbohydrate and crude protein, J. Dairy Sci. 73 (1990) 1039-1050.

[5] Chalmers J.S., Thomas P.C., Chamberlain D.G., The effect of intraruminal infusions of propionic acid on milk composition in cows given silage diets, Proc. Nutr. Soc. 39 (1980) $27 \mathrm{~A}$.

[6] Clark J.H., Spires H.R., Derrig R.G., Bennink M.R., Milk production, nitrogen utilization and glucose synthesis in lactating cows infused postruminally with sodium caseinate and glucose, J. Nutr. 107 (1977) 631-644.

[7] Coulon J.B., Rémond B., Variations in milk output and milk protein content in response to the level of energy supply to the dairy cow: a review, Livest. Prod. Sci. 29 (1991) 31-47.

[8] Dhiman T.R., Cadorniga C., Satter L.D., Protein and energy supplementation of high alfalfa silage diets during early lactation, $\mathbf{J}$. Dairy Sci. 76 (1993) 1945-1959.

[9] Emery R.S., Feeding for increased milk protein, J. Dairy Sci. 61 (1978) 825-828.

[10] Fisher L.J., Elliot J.M., Effect of intravenous infusion of propionate or glucose on bovine milk composition, J. Dairy Sci. 49 (1966) 826-828

[11] Frobish R.A., Davis C.L., Effects of abomasal infusions of glucose and propionate on milk yield and composition, J. Dairy Sci. 60 (1977) 204-209.

[12] Guinard J., Rulquin H., Vérité R., Effect of graded levels of duodenal infusions of casein on mammary uptake in lactating cows. 1. Major constituents, J. Dairy Sci. 77 (1994) 2221-2231.

[13] Holter J.B., Jones L.A., Colovos N.F., Urban W.E. Jr., Caloric value of acetate and propionate for lactating dairy cows, J. Dairy Sci. 55 (1972) 1757-1762.

[14] Hurtaud C., Rulquin H., Vérité R,, Effect of infused volatile fatty acids and caseinate on milk composition and coagulation in dairy cows, J. Dairy Sci. 76 (1993) 301 1-3020.

[15] Hurtaud C., Rulquin H., Delaite M., Vérité R., Appréciation de l'aptitude fromagère des laits de vaches individuels. Tests d'aptitude fromagère et rendement fromager de fabrication, Lait 44 (1995) 385-398.

[16] Inra, Utilisation énergétique des produits terminaux de la digestion, in: Jarrige R. (Ed.), Alimentation des ruminants, Inra publications, Versailles, France, 1978, pp. 47-88.
[17] Inra, Introduction. Feeding standards for ruminants, in: Jarrige R. (Ed.), Ruminant Nutrtion. Recommended Allowances and Feed Tables, John Libbey, Eurotext, London, UK, 1989, pp. 15-22.

[18] Jouany J.P., Volatile fatty acid and alcohol determination in digestive contents, silage juices, bacterial cultures and anaerobic fermentor contents, Sci. Aliments 2 (1982) 131-144.

[19] Journet M., Chilliard Y., Influence de l'alimentation sur la composition du lait. 1. Taux butyreux: facteurs généraux, Bull. Tech. Inst. Natl. Rech. Agron. CRZV 60 (1985) 13-23.

[20] Judson G.J., Leng R.A., Studies on the control of gluconeogenesis in sheep: effect of propionate, casein and butyrate infusions, $\mathrm{Br}$. J. Nutr. 29 (1973) 175-195.

[21] Kennelly J.J., The biological potential to alter the composition of cow's milk, in: Ivan $\mathrm{M}$. (Ed.), Animal Science Research and Development: Moving Toward a New Century, Centre for Food and Animal Research, Agriculture and Agri-Food Canada, Ottawa, ON Canada, 1995, pp. 61-78.

[22] Kreikemeier K.K., Harmon D.L., Abomasal glucose, maize starch and maize dextrin infusions in cattle: small-intestinal disappearance, net portal flux and ileal oligosaccharide flow, Br. J. Nutr. 73 (1995) 763-772.

[23] Kreikemeier K.K., Harmon D.L., Brandt R.T.J., Avery T.B., Johnson D.E., Small intestinal starch digestion in steers: effect of various levels of abomasal glucose, corn starch and corn dextrin infusion on small intestinal disappearance and net glucose absorption, J. Anim. Sci. 69 (1991) 328-338.

[24] Lemosquet S., Rideau N., Rulquin H., Faverdin P., Simon J., Vérité R., Effects of a duodenal glucose infusion on the relationship between plasma concentration of glucose and insulin in dairy cows, J. Dairy Sci. 80 (1997) 2854-2865.

[25] Massart-Leën A.M., Roets E., Peeters G., Verbeke R., Propionate for fatty acid synthesis by the mammary gland of the lactating goat, J. Dairy Sci. 66 (1983) 1445-1454.

[26] Moore J.H., Christie W.W., Lipid metabolism in the mammary gland of ruminant animals, Prog. Lipid. Res. 17 (1979) 347-395.

[27] Oldick B.S., Staples C.R., Thatcher W.W., Gyawu P., Abomasal infusion of glucose and fat-Effect on digestion, production, and ovarian and uterine functions of cows, J. Dairy Sci. 80 (1997) 1315-1328.

[28] Ørskov E.R., Flatt W.P., Moe P.W., Munson A.W., The influence of ruminal infusion of volatile fatty acids on milk yield and composition and on energy utilization by lactating cows, Br. J. Nutr. 23 (1969) 443-453. 
[29] Ørskov E.R., Grubb D.A., Kay R.N., Effect of postruminal glucose or protein supplementation on milk yield and composition in Friesian cows in early lactation and negative energy balance, Br. J. Nutr. 38 (1977) 397-405.

[30] Peyraud J.L., Widyobroto B.P., Effect of the nature and the rate of ruminal degradation of carbohydrates on site and extent of digestion in dairy cows, Proc. Soc. Nutr. Physiol. 3 (1994) 34.

[31] Remeuf F., Cossin V., Dervin C., Lenoir J., Tomassone R., Relations entre les caractères physico-chimiques des laits et leur aptitude fromagère, Lait 71 (1991) 397-421.

[32] Rémond B., Influence de l'alimentation sur la composition du lait de vache. 2. Taux protéique : facteurs généraux, Bull. Tech. Inst. Natl. Rech. Agron. CRZV 62 (1985) 53-67.

[33] Rook J.A.F., Balch C.C., The effects of intraruminal infusions of acetic, propionic and butyric acids on the yield of milk and composition of the milk of the cow, Br. J. Nutr. 15 (1961) 361-369.

[34] Rook J.A.F., Balch C.C., Johnson V.W., Further observations on the effects of intraruminal infusions of volatile fatty acids and of lactic acid on the yield and composition of the milk of the cow, Br. J. Nutr. 19 (1965) 93-99.

[35] Rulquin H., Effets sur la digestion et le métabolisme des vaches laitières d'infusions d'acides gras volatils dans le rumen et de caséinate dans le duodénum. I. Production et digestion, Reprod. Nutr. Dev. 22 (1982) 905-921.

[36] Rulquin H., Hurtaud C., Nutrition de la vache laitière et composition du lait, in : Symposium sur les bovins laitiers. Atteindre l'équilibre, Conseil des Productions Animales du
Québec, Ministère de l'Agriculture, des Pêcheries et de l'Alimentation, Québec, QC, Canada, 1993, pp. 123-137.

[37] $S A S^{\circledast}$ User's Guide: Statistics, Version 6 Edition, SAS Inst., Inc., Cary, NC, 1987.

[38] Seal C.J., Parker D.S., Effect of intraruminal propionic acid infusion on metabolism of mesenteric- and portal-drained viscera in growing steers fed a forage diet: I. Volatile fatty acids, glucose, and lactate, J. Anim. Sci. 72 (1994) 1325-1334.

[39] Spörndly E., Effects of diet on milk composition and yield of dairy cows with special emphasis on milk protein content, Swedish J. Agric. Res. 19 (1989) 99-106.

[40] Thomas P.C., Martin P.A., The influence of nutrient balance on milk yield and composition, in: Garnsworthy P.C. (Ed.), Nutrition and Lactation in the Dairy Cow, Butterworths, London, UK, 1988, pp. 97-118.

[41] Vik-Mo L., Emery R.S., Huber J.T., Milk protein production in cows abomasally infused with casein or glucose, J. Dairy Sci. 57 (1974) 869-877.

[42] Wilson G.F., Davey A.W.F., Dolby R.M., Milk composition as affected by intra-ruminal infusion of volatile fatty acids to cows on a restricted ration, NZ J. Agric. Res. 10 (1967) 215-225.

[43] Whitelaw F.G., Milne J.S., Ørskov E.R., Smith J.S., The nitrogen and energy metabolism of lactating cows given abomasal inusions of casein, Br. J. Nutr. 55 (1986) 537-556.

[44] Zannoni M., Annibaldi M., Standardization of the renneting ability of milk by Formagraph, Sci. Tec. Latt. Casearia 32 (1981) 79 94. 